\title{
๖Mountain Waves Produced by a Stratified Shear Flow with a Boundary Layer. Part II: Form Drag, Wave Drag, and Transition from Downstream Sheltering to Upstream Blocking
}

\author{
François LotT, ${ }^{\mathrm{a}}$ BRuno DeRemble, ${ }^{\mathrm{a}}$ AND ClÉment SOUFFLET ${ }^{\mathrm{a}}$ \\ ${ }^{\text {a }}$ Laboratoire de Météorologie Dynamique, PSL Research Institute, Ecole Normale Supérieure, Paris, France
}

(Manuscript received 15 May 2020, in final form 10 September 2020)

\begin{abstract}
The nonhydrostatic version of the mountain flow theory presented in Part I is detailed. In the near-neutral case, the surface pressure decreases when the flow crosses the mountain to balance an increase in surface friction along the ground. This produces a form drag that can be predicted qualitatively. When stratification increases, internal waves start to control the dynamics and the drag is due to upward-propagating mountain waves as in Part I. The reflected waves nevertheless add complexity to the transition. First, when stability increases, upward-propagating waves and reflected waves interact destructively and low-drag states occur. When stability increases further, the interaction becomes constructive and high-drag states are reached. In very stable cases, the reflected waves do not affect the drag much. Although the drag gives a reasonable estimate of the Reynolds stress, its sign and vertical profile are profoundly affected by stability. In the nearneutral case, the Reynolds stress in the flow is positive, with a maximum around the top of the inner layer, decelerating the large-scale flow in the inner layer and accelerating it above. In the more stable cases, on the contrary, the large-scale flow above the inner layer is decelerated as expected for dissipated mountain waves. The structure of the flow around the mountain is also strongly affected by stability: it is characterized by nonseparated sheltering in the near-neutral cases, by upstream blocking in the very stable case, and at intermediate stability by the presence of a strong but isolated wave crest immediately downstream of the ridge.
\end{abstract}

KEYWORDS: Atmosphere; Downslope winds; Gravity waves; Mountain waves; Boundary layer; Mountain meteorology

\section{Introduction}

The impact of small- to medium-scale mountains on atmospheric dynamics is extremely sensitive to the stratification. In neutral flows, the atmospheric boundary layer stress changes the flow and hence the surface pressure on either side of the mountain. This produces a form drag that will in turn drive an exchange of momentum between the atmosphere and the Earth's surface (Hunt et al. 1988). This pressure drop on the lee side is associated with an effect of downstream sheltering. For obstacles with small slopes, the sheltering is nonseparated, but for obstacles with larger slopes, this sheltering is separated (Reinert et al. 2007) and can cause the formation of banner clouds (Voigt and Wirth 2013). The dynamical regime in the stably stratified case is fundamentally different because internal gravity waves create a drag even in the absence of boundary layer (Durran 1990). For small mountains, the asymmetry in the fields near the surface is such that the flow decelerates upstream, and it accelerates downstream. This can cause a form of nonseparated upstream blocking with strong downslope winds (Lott et al. 2020, hereafter Part I). For large mountains, the situation is different because the associated waves approach breaking, a dynamics that produces separated upstream blocking and strong downslope winds [see recent examples in Pokharel et al. (2017)]. To summarize and from a qualitative point of view, two radically different flow regimes

¿ Denotes content that is immediately available upon publication as open access.

Corresponding author: François Lott, flott@lmd.ens.fr occur above a mountain: on the one hand, we assist to the development of strong upslope winds in neutral case, and on the other hand, we see strong downslope winds in the stratified case.

Although the two type of dynamics in the neutral and stratified case are today quite well understood, it remains unclear what parameter characterizes the transition between the two regimes. For small mountains, the seminal paper of Belcher and Wood (1996) describes a transition from form drag to wave drag that occurs when the Froude number $F_{m}=$ $U\left(h_{m}\right) / N\left(h_{m}\right) / L \approx 1$ (with $U$ the incident flow velocity, and $N$ the Brunt-Väisälä frequency measured at a midlayer height $h_{m}$; see henceforth). When the Froude number $F_{m}<1$, the dynamics is neutral and the drag is a form drag, but when $F_{m}>$ 1 this form drag is replaced by a wave drag. Belcher and Wood (1996) also shows that the wave drag is that predicted by inviscid theory, if we take for incident flow parameters those at the midlayer height $h_{m}$, an altitude where the disturbance dynamics is inviscid and largely controlled by the curvature of the background wind. Mathematically, for a mountain of characteristic horizontal scale $L, h_{m}$ is the altitude where the ray of curvature of the background wind equals $L$ :

$$
\frac{u_{0}\left(h_{m}\right)}{u_{0 z z}\left(h_{m}\right)}=L^{2},
$$

$u_{0}$ and $u_{0 z z}$ being the background wind and its second vertical derivative, respectively. While Belcher and Wood (1996) do not describe the transition in terms of upstream/downstream separation (upstream separation indicating blocking), the theoretical analysis of Ambaum and Marshall (2005) shows that neutral flows separate on the lee side and that this 
separation is largely inhibited in the stable case, or, more precisely, that it occurs much farther on the lee side beyond the first lee-wave trough and upstream the mountain crest. This leeside separation and, more generally, the interaction between the boundary layer and mountain waves are central in the development of downstream rotors (Doyle and Durran 2002; Sachsperger et al. 2016).

Maybe because early theories on boundary layer flow over mountains demand quite involved asymptotic analysis (Belcher and Wood 1996), subsequent theories on the interactions between boundary layer and mountain have often used simplified representation of the boundary layer to remain tractable (Smith et al. 2006; Lott 2007). To a certain extent, these simplifications mirror the simplifications made in the literature on stable boundary layer over complex terrain. In such studies, the inviscid dynamics often boils down to that above the boundary layer all the mountain waves propagate upward without being reflected back (Belcher and Wood 1996; Weng 1997; Athanassiadou 2003). There is nevertheless a growing effort in the community to analyze the interaction between boundary layers and mountain waves (Tsiringakis et al. 2017; Lapworth and Osborne 2019). These efforts are motivated by the fact that present day numerical weather prediction and climate models still make errors in the representation of subgrid-scale orography (SSO) and because these errors are at scales where neutral dynamics and stratified dynamics can no longer be treated separately [see discussion in Serafin et al. (2018) and in Part I]. Also, a remaining issue in SSO parameterizations still concerns the representation of the vertical distribution of the wave Reynolds stress (Tsiringakis et al. 2017; Lapworth and Osborne 2019), and existing theories do not tell much about this.

To better understand this vertical distribution, we argued in Part I that the theory in the simplest case with constant eddy viscosity $\nu$ needed to be developed beyond the historical papers [see Smith (1973) for the neutral case and Sykes (1978) for the stratified case]. In fact, we showed in Part I that with constant viscosity, we were able to predict the wave field with uniform approximation over the entire domain. This permits us to calculate altogether the mountain drag, the wave Reynolds stress vertical profile, and the nonseparated structure of the flow within the boundary layer (in the form of upstream blocking and downslope winds). Using these solutions, we showed that the wave pressure drag and stress can be deduced from mountain-wave linear theory if we evaluate the background flow at the "inner layer" scale,

$$
\delta=\left(\frac{\nu L}{u_{0 z}}\right)^{1 / 3}
$$

with $u_{0 z}$ the background wind shear. We insist that this inner scale is distinct from a boundary layer height, the latter being infinite in the constant shear case. In Part I, we also showed that the wave Reynolds stress that radiates aloft the inner layer (which total depth is estimated around $5 \delta$ ) is only a fraction of the surface pressure drag: internal waves are substantially dissipated when they travel through the inner layer and part of the wave drag is deposited near the top of the inner layer. Last, we showed in Part I that for mountains with height $H \ll \delta$, the wave stress is extracted from the boundary layer rather than from the surface as in the inviscid case. This means that the interaction between the boundary layer and the obstacle accelerates the large-scale flow near the surface as waves are emitted. Finally, for mountain with height $H \approx \delta$, we showed that upstream blocking and downslope winds occur within the boundary layer. Because we built our analysis on linear dynamics, these phenomena correspond to nonseparated dynamics by construction. They actually mirror the nonseparated intensified upslope winds and downstream sheltering that occurs in the neutral case.

A first limit of Part I is that we only considered upwardpropagating internal waves above the inner layer. This is a serious limitation, reflected waves potentially affecting the boundary layer when they return to the ground. A second limit is that we only studied constant shear within the hydrostatic approximation. In this situation, the properties of the inviscid solution make that we cannot study weakly stratified situations and analyze the transition from neutral to stratified flows.

The purpose of the present paper is, therefore, to work with a nonhydrostatic model in order to analyze the case where all the harmonics are reflected. As we shall see in section 2, this happens with constant infinite shear in the nonhydrostatic Boussinesq approximation. In section 3, we describe a characteristic wave field and extend the mountain-wave drag predictor proposed in Part I to the neutral case. We demonstrate that we need to substitute it by a form drag for small values of the Richardson number $(J<1)$. We analyze the transition from neutral to stratified situation for small slopes in section 4 and show that reflected waves can interact destructively or constructively with the surface when $J \approx 1$ yielding low-drag and high-drag states. We then analyze in section 5 the action of the waves on the large-scale flow and show that this action differs between the neutral cases and the stratified cases. In section 6 , we describe situations with slopes comparable to the inner-layer scale. In this case neutral flows are characterized by strong upslope winds and nonseparated sheltering on the lee side, whereas in stable case we recover the strong downslope winds and upstream blocking found in Part. I. All our results have been validated with the full nonlinear model used in Part I, the results of which are mentioned throughout the paper. We conclude and present perspectives in section 6 .

\section{Theory}

Many elements are reminiscent of Part I, so we recall in this section the general formulation and only emphasize the differences. As in Part I, we consider a background flow with constant shear $u_{0 z}$ and constant stratification $\rho_{0 z}$

$$
u_{0}(z)=u_{0 z} z ; \quad \rho_{0}(z)=\rho_{r}+\rho_{0 z} z
$$

incident on a Gaussian ridge of maximum height $H$ and characteristic length $L$ :

$$
h(x)=H e^{-x^{2} /\left(2 L^{2}\right)} .
$$


We then consider obstacles well embedded into the "inner" layer and use linear equations that we normalize by introducing the "outer" scaling:

$$
\begin{aligned}
(x, z) & =L(\bar{x}, \bar{z}), \quad\left(u^{\prime}, w^{\prime}\right)=u_{0 z} L(\bar{u}, \bar{w}), \\
\left(p^{\prime}, b^{\prime}\right) & =\left(\rho_{r} u_{0 z}^{2} L^{2} \bar{p}, u_{0 z}^{2} L \bar{b}\right),
\end{aligned}
$$

where the primes are for disturbances and the overbars for dimensionless variables. All notations are standard: $x, z, u^{\prime}$, and $w^{\prime}$ have their conventional definitions, and $b^{\prime}$ is the disturbance buoyancy. The relevant nondimensional parameters are

$$
J=-\frac{g \rho_{0 z}}{\rho_{r} u_{0 z}^{2}}, \quad P=\frac{\nu}{\kappa}, \quad S=\frac{H}{L}, \quad \text { and } \quad \bar{\nu}=\frac{\nu}{u_{0 z} L^{2}}
$$

where $J$ is a Richardson number, $P$ is a Prandtl number, $S$ is a slope parameter, and $\bar{\nu}$ is an inverse Reynolds number. Henceforth, we only work with nondimensional variables, and the stationary 2D Boussinesq linear equations we use are as in Part I except that the hydrostatic approximation [Eq. (5) in Part I] is replaced by the equation for the vertical acceleration:

$$
\bar{z} \partial_{\bar{x}} \bar{w}=-\partial_{\bar{z}} \bar{p}+\bar{b}+\bar{\nu} \partial_{\bar{z}}^{2} \bar{w}
$$

At the topography, we use the three boundary conditions:

$$
\bar{h}(\bar{x})+\bar{u}(\bar{x}, \bar{h})=\bar{w}(\bar{x}, \bar{h})=J \bar{h}(\bar{x})+\bar{b}(\bar{x}, \bar{h})=0 \quad \text { at } \quad \bar{h}=S e^{-x^{2} / 2}
$$

The Boussinesq equations satisfy a wave action budget that is slightly different than in the hydrostatic case:

$$
\begin{aligned}
& \frac{\partial}{\partial \bar{x}} \underbrace{(\underbrace{\bar{z} \frac{\partial_{\bar{z}} \bar{u}-\partial_{\bar{x}} \bar{w}}{J} \bar{b}}_{A}+\frac{\bar{b}^{2}}{2 J}+\frac{\bar{u}^{2}-\bar{w}^{2}}{2})}_{F^{x}}+\frac{\partial}{\partial \bar{z}} \underbrace{\overline{u w}}_{F^{z}} \\
& =\underbrace{\frac{\bar{\nu}}{\bar{b}} \partial_{\bar{z}}^{2}\left(\partial_{\bar{z}} \bar{u}-\partial_{\bar{x}} \bar{w}\right)+P^{-1} \frac{\bar{\nu}}{J}\left(\partial_{\bar{z}} \bar{u}-\partial_{\bar{x}} \bar{w}\right) \partial_{\bar{z}}^{2} \bar{b}}_{Q},
\end{aligned}
$$

where $A$ is the pseudomomentum, $F^{x}$ and $F^{z}$ the horizontal and vertical components of the pseudomomentum flux, and $Q$ its production/destruction by dissipative processes.

\section{a. Outer solution}

We then search inflow solutions in term of Fourier transform, and for high Reynolds number $(\bar{\nu} \ll 1)$, the dynamics is inviscid at leading order. In this case the Fourier transform of the vertical velocity, $\overline{\mathbf{w}}(\bar{k}, \bar{z})$, is solution of Bessels's equation,

$$
\overline{\mathbf{w}}_{\overline{z z}}+\left(\frac{J}{\bar{z}^{2}}-\bar{k}^{2}\right) \overline{\mathbf{w}}=0 .
$$

When the horizontal wavenumber $\bar{k}>0$, and $J>1 / 4$ a bounded solution in $\bar{z} \rightarrow \infty$ can be expressed in terms of the Hankel function,

$$
\overline{\mathbf{w}}_{I}(\bar{k}, \bar{z})=i \sqrt{\frac{\pi \bar{k} \bar{z}}{2}} e^{-\mu \pi / 2} H_{i \mu}^{(1)}(i \bar{k} \bar{z}), \quad \text { where } \quad \mu=\sqrt{\left|J-\frac{1}{4}\right|} .
$$

A first major difference with the hydrostatic case is that we can now treat the weakly stratified situations when $J<1 / 4$ simply by changing $\mu$ in $i \mu$. Also, in (11) we introduce the notation $\overline{\mathbf{w}}_{I}$ to indicate that we choose a particular inviscid solution that is scaled to behave like an exponentially decaying solution of "unit" amplitude in the far field [see (9.2.3) in Abramowitz and Stegun (1964)]:

$$
\overline{\mathbf{w}}_{I}(\bar{k}, \bar{z}) \underset{\bar{z} \rightarrow \infty}{\approx} e^{-\bar{k} \bar{z}} .
$$

This also shows that all harmonics are trapped, which is another major difference compared to the hydrostatic case (in the latter case, all waves propagate upward without reflection). From this and the limiting form of the Hankel functions when $\bar{z} \rightarrow 0$ [see (9.1.9) in Abramowitz and Stegun (1964)] we write the asymptotic form of the inviscid solution near the surface as

$$
\begin{aligned}
\overline{\mathbf{w}}_{I}(\bar{k}, \bar{z}) \underset{\bar{z} \rightarrow 0}{\approx} \overline{\mathbf{w}}_{M}(\bar{k}, \bar{z}) & =\bar{a}_{1}(\bar{k}) \bar{z}^{1 / 2-i \mu}+\bar{a}_{2}(\bar{k}) \bar{z}^{1 / 2+i \mu}, \text { with } \\
\bar{a}_{1}(\bar{k}) & =-\frac{i \sqrt{\pi}}{\sinh (\mu \pi) \Gamma(1-i \mu)}(\bar{k})^{1 / 2-i \mu}, \\
\bar{a}_{2}(\bar{k}) & =\bar{a}_{1}(\bar{k})^{*} .
\end{aligned}
$$

\section{b. Inner solutions}

To get the solutions in the inner layer, we introduce the scaling

$$
\begin{aligned}
& \bar{z}=\bar{\delta} \tilde{z}, \quad(\overline{\mathbf{w}}, \overline{\mathbf{w}})=(\tilde{\mathbf{u}}, \bar{\delta} \bar{k} \tilde{\mathbf{w}}), \quad(\bar{p}, \bar{b})=(\bar{\delta} \tilde{p}, \tilde{b}), \quad \text { where } \\
& \bar{\delta}=\left(\frac{\bar{\nu}}{\bar{k}}\right)^{1 / 3} .
\end{aligned}
$$

At leading order and with this scaling, the inner-layer equations are as in Part I [Eq. (16)] they can be reduced to a sixth-order equation for $\tilde{\mathbf{w}}$ [Part I, Eq. (17)]. Among its six independent solutions, only the three with asymptotic form in $\tilde{z} \gg 1$,

$$
\begin{aligned}
\tilde{\mathbf{w}}_{12} & \approx \tilde{a}_{1}(\bar{k}) \tilde{z}^{1 / 2-i \mu}+\tilde{a}_{2}(\bar{k}) \tilde{z}^{1 / 2+i \mu} \\
\tilde{\mathbf{w}}_{3} & \approx \tilde{z}^{-5 / 4} e^{-(2 \sqrt{i} / 3) \tilde{z}^{3 / 2}}, \quad \tilde{\mathbf{w}}_{4} \approx \tilde{z}^{-9 / 4} e^{-(2 \sqrt{i P} / 3) \tilde{z}^{3 / 2}} .
\end{aligned}
$$

need to be considered. As in Part I, they are evaluated numerically and the matching with the outer layer is simply done by taking

$$
\tilde{a}_{1}(\bar{k})=\frac{\bar{a}_{1}}{\bar{k}} \bar{\delta}^{-1 / 2-i \mu}, \quad \tilde{a}_{2}(\bar{k})=\tilde{a}_{1}(\bar{k})^{*} .
$$

This guarantees that $\tilde{\mathbf{w}}_{12}$ matches the inviscid solution $\overline{\mathbf{w}}_{I}$ according to (13) and (15).

Next, we assume that the mountain is well in the inner layer and use the inner solution to satisfy the lower 

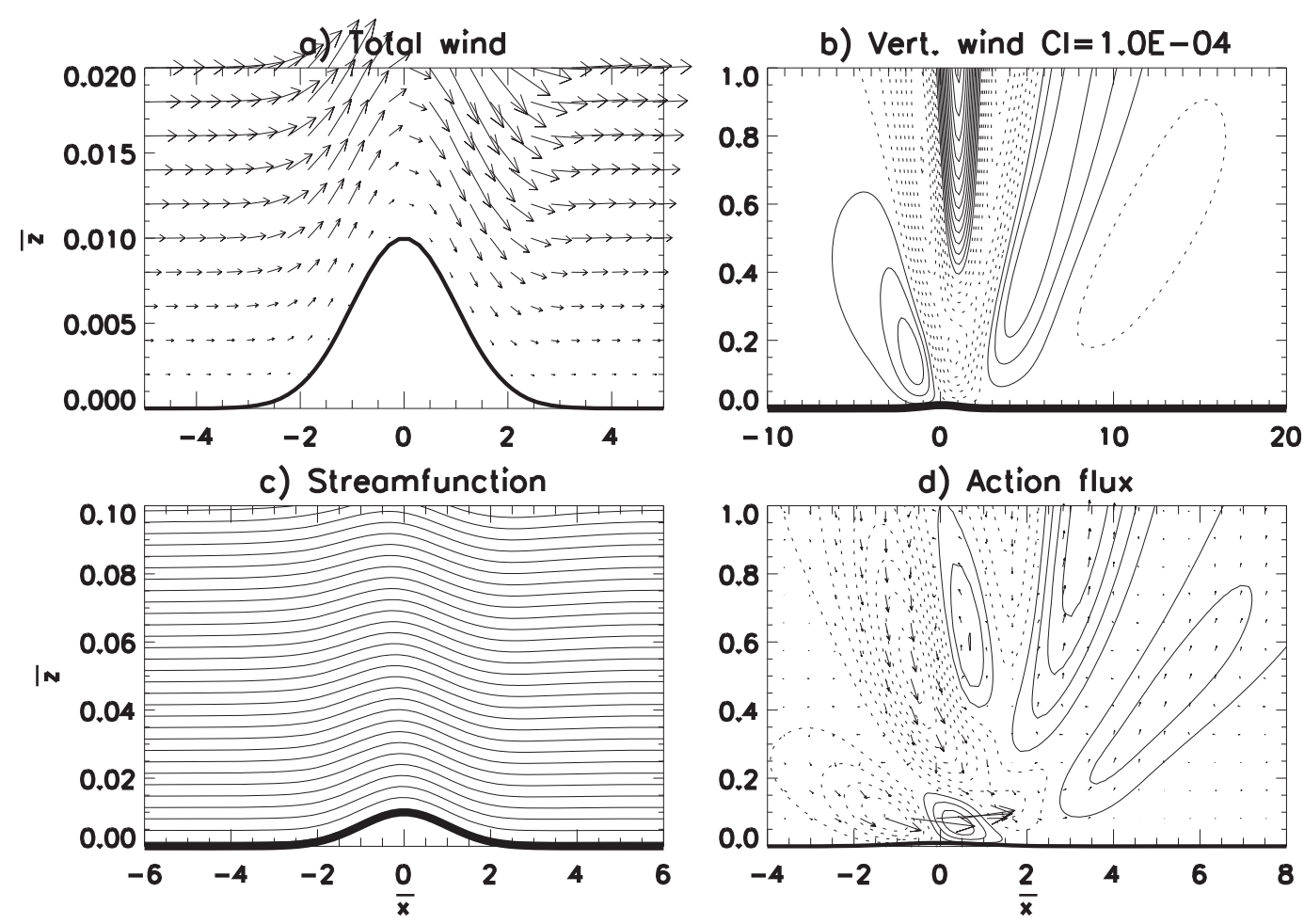

FIG. 1. Physical fields predicted by the viscous theory when $J=4, S=0.01$, and $\bar{\delta}=0.1$. (a) Total wind vector $(\bar{z}+\bar{u}, \bar{w})$. (b) Vertical wind $\bar{w}$. (c) Total streamfunction $\bar{\psi}$ defined by $\partial_{\bar{z}} \bar{\psi}=\bar{z}+\bar{u}$. (d) Vertical flux of action $F^{z}$ and action flux vector $\left(F^{x}, F^{z}\right)$. In (b) and (d), the negative values are dashed.

boundary conditions (8). The equation for the vertical velocity is written

$$
\begin{aligned}
\bar{w}(\bar{x}, \bar{h}) \approx & \int_{-\infty}^{+\infty} \bar{k} \bar{\delta}(\bar{k})\left[f_{12}(\bar{k}) \tilde{\mathbf{w}}_{12}(\bar{k}, \tilde{h})+f_{3}(\bar{k}) \tilde{\mathbf{w}}_{3}(\tilde{h})\right. \\
& \left.+f_{4}(\bar{k}) \tilde{\mathbf{w}}_{4}(\tilde{h})\right] e^{i \bar{k} \bar{x}} d \bar{k}=0
\end{aligned}
$$

where $\tilde{h}(\bar{x}, \bar{k})=\bar{h}(\bar{x}) / \bar{\delta}(\bar{k})$. Inversion of this integral equation together with the two integral equations expressing the boundary conditions on $\bar{u}(h)$ and $\bar{b}(h)$ permits us to evaluate $f_{12}(\bar{k}), f_{3}(\bar{k})$, and $f_{4}(\bar{k})$.

Since we are now in the presence of an exact inviscid solution connected to a viscous solution via the matching function $\tilde{\mathbf{w}}_{M}(\bar{k}, \bar{z})$ we can follow conventional techniques to build a uniform approximation. To do so, we express the viscous solution in terms of the outer variables, for example, by writing

$$
\begin{aligned}
\overline{\mathbf{w}}_{V}(\bar{k}, \bar{z})= & \bar{k} \bar{\delta}(k)\left\{f_{12}(\bar{k}) \tilde{\mathbf{w}}_{12}[\bar{k}, \bar{z} / \bar{\delta}(\bar{k})]\right. \\
& +f_{3}[(\bar{k}), \bar{z} / \bar{\delta}(\bar{k})] \tilde{\mathbf{w}}_{3}[\bar{k}, \bar{z} / \bar{\delta}(\bar{k})] \\
& \left.+\tilde{\mathbf{w}}_{4}(\bar{k}, \bar{z}) \tilde{\mathbf{w}}_{4}[\bar{k}, \bar{z} / \bar{\delta}(\bar{k})]\right\}
\end{aligned}
$$

and use for $\overline{\mathbf{w}}(\bar{k}, \bar{z})$ the uniform approximation,

$$
\overline{\mathbf{w}}(\bar{k}, \bar{z})=f_{12}\left[\overline{\mathbf{w}}_{I}(\bar{k}, \bar{z})-\overline{\mathbf{w}}_{M}(\bar{k}, \bar{z})\right]+\overline{\mathbf{w}}_{V}(\bar{k}, \bar{z}),
$$

again with similar expression for the horizontal wind and buoyancy.
The solutions used in the following are then obtained via inverse Fourier transform of the uniform approximations, and as in Part I, we validate these solutions with nonlinear simulations done with the MITgcm (Marshall et al. 1997). The configuration of this model is essentially the same as in Part I except that we run it in nonhydrostatic mode. All the $2 \mathrm{D}$ fields (winds, buoyancy, streamfunction) from this model are essentially the same as from the linear model so we will only plot 2D fields from the linear model.

\section{Transition from form drag to wave drag}

In Fig. 1 we plot the flow response when the slope parameter $S=0.01$, is much smaller than the inner-layer scale $\bar{\delta}(1)=0.1$ and the Richardson number $J=4$. We also take a Prandtl number $P=0.5$, that will stay unchanged in the remainder of the analysis. Henceforth, we will call this case the reference case. Note that these values are the same as in Part I to allow us direct comparison between Fig. 1 here and its hydrostatic counterpart (Fig. 1 of Part I).

The total wind at low level in Fig. 1a contours well the obstacle and is null at the surface as expected. We plot in Fig. 1b the vertical velocity field that highlights a system of gravity waves. In the upstream region $x<0$, the phase lines tilt against the shear indicating upward propagation, directly above the hill the wave phase lines are more vertical, and downstream they become tilted in the direction of the shear indicating downward propagation. Such structure suggests that the mountain 
produces upward-propagating gravity waves, that these waves are entirely reflected in the far field (the waves phase lines tilt downstream is almost symmetric and opposite to their upstream tilt) and are almost entirely absorbed when they return to the surface (the wave amplitude rapidly decreases when horizontal distance increases). It is important to note that the amplitude of the vertical velocity is of the same order of magnitude as the amplitude predicted in Part I, which is the amplitude predicted by linear theory if we take for the incident wind at the ground the average of the incident wind over the inner-layer scale $(\bar{\delta}(1) / 2)$. In Part I, we interpreted that by the fact that over a distance equal to the inner-layer scale, the viscous dynamics produces a flow that streamlines have vertical displacements with amplitude near the mountain height (as we see here in Fig. 1c), as a consequence, the waves produced by the inner layer resemble the inviscid waves produced by a lower boundary located at $\bar{h}(\bar{x})+\bar{\delta}$.

Finally, the wave action flux in Fig. 1d confirms that the waves are produced indirectly by the distortion of the inner layer rather than directly by the mountain (the wave action flux in the inner layer is oriented from one side of the mountain to the other). The orientation of the wave action flux aloft the inner layer also corroborates the fact that over the obstacle the waves propagate upward (the wave action flux points toward the surface), whereas the wave field downstream is dominated by downward-propagating wave (the wave action flux is everywhere pointing upward, $F^{z}>0$ ). The fact that $F^{z}>0$ almost everywhere on the lee side is also consistent with the fact that there is almost no surface reflection on the ground. This contrasts with Part I, where downward waves were excluded by construction, such that in the hydrostatic case, we had $F^{z}<0$ almost everywhere above the inner layer (see Fig. 1d in Part I).

In Part I, we noticed that predicting the wave amplitude with linear inviscid theory was also useful to scale the mountain waves stress and drag,

$\overline{\bar{u}} \bar{w}(\bar{z})=\int_{-\infty}^{+\infty} \bar{u}(\bar{x}, \bar{z}) \bar{w}(\bar{x}, \bar{z}) d \bar{x}, \quad \operatorname{Dr}=-\int_{-\infty}^{+\infty} \bar{p}(\bar{x}, \bar{h}) \frac{\partial \bar{h}}{\partial \bar{x}} d \bar{x}$,

More precisely, we found that the predictor

$$
\operatorname{Dr}_{\mathrm{GWP}}=\sqrt{J} \bar{\delta}(1) S^{2} / 2
$$

provides a good description of the drag for a large range of slopes $S$ and for Richardson numbers $J>0.25$. This scaling was, however, based on hydrostatic theory, such that we cannot use it for neutral cases $(J \ll 1)$. In neutral cases, the mountain drag becomes a form drag due to dissipative loss of pressure when the air passes over the obstacle. To estimate this drag we next make the conventional hypothesis that in the inner layer the pressure varies little in the vertical direction and that the horizontal pressure gradient balances the divergence of the viscous stress,

$$
\partial_{\bar{x}} \bar{p} \approx \bar{\nu} \partial_{\bar{z}}^{2} \bar{u}
$$

If we then remark that in the inner layer the wind increases from 0 (at the surface) to $\bar{h}$ (at the top of the inner layer), then the surface wind shear should be on the order of $\bar{h} / \bar{\delta}(1)$. We can then estimate the form drag as a vertical integral of (23)

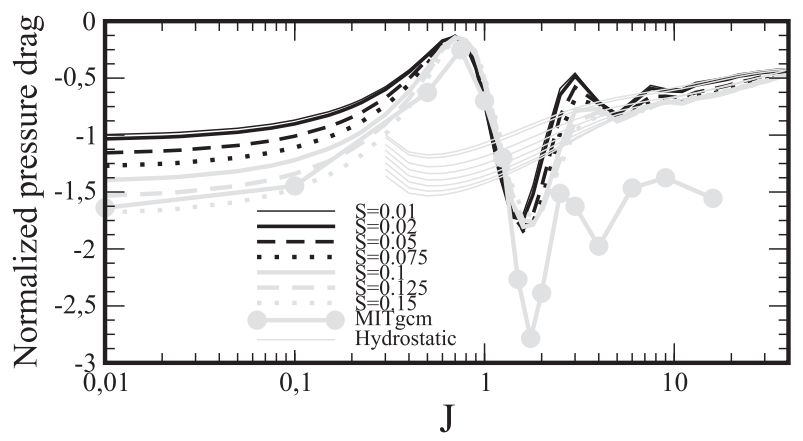

FIG. 2. Surface pressure drag normalized by the predictor $\operatorname{Dr}_{p}$ in (26). The hydrostatic pressure drag normalized by $\operatorname{Dr}_{\mathrm{GWP}}$ from Part I is also shown for comparison (thin gray lines). The gray dots are from the MITgcm with $S=0.15$.

over the inner layer. We get $\bar{\delta}(1) \partial_{\bar{x}} \bar{p} \approx-\bar{\nu} \bar{h} / \bar{\delta}(1)=-\bar{\delta}(1)^{2} \bar{h}$. We can thus estimate the form drag as

$$
\int_{-\infty}^{+\infty} \bar{h} \partial_{\bar{x}} \bar{p} d \bar{x} \approx-\int_{-\infty}^{+\infty} \bar{\delta}(1) \bar{h}^{2}=-\sqrt{\pi} \bar{\delta}(1) S^{2} .
$$

Because this evaluation is qualitative and because the transition between stratified cases and near-neutral cases is more likely occurring near $J=1$ we simplify the form drag predictor in

$$
\operatorname{Dr}_{\mathrm{FDP}}=\bar{\delta}(1) \frac{S^{2}}{2}
$$

Then, following Belcher and Wood (1996) we take as predictor of the mountain drag and stress the maximum between (22) and (25):

$$
\operatorname{Dr}_{P}=\operatorname{Max}(1, \sqrt{J}) \bar{\delta}(1) \frac{S^{2}}{2} .
$$

We plot in Fig. 2 the mountain drag normalized by this predictor for several values of $J$ and $S$. We see that the predictor is quite accurate (the ratio is around 1) at least when the flow is stable $(J>3)$ or neutral $(J<0.1)$. But there is a transition zone when $J \approx 1$, which seems quite rich dynamically. This transition is characterized by a relative maximum of the drag near $J=1.6$, and a relative minimum near $J=0.7$ that were completely absent in the hydrostatic case [see the thin gray lines in Fig. 2 and remember again that, in Part I, (i) the cases with $J<0.25$ were not treated, and (ii) that the reflected waves were absent by construction]. To understand the physics behind the minimum and maximum values of the drag for intermediate values of $J$, it is important to include the reflected waves in the discussion. We recall that the altitude of dominant turning point of the wave field, which is the turning point above which the dominant wavenumber $\bar{k}=1$ becomes evanescent is $\bar{z}_{T}(1)=\sqrt{J}$ and so increases with $J$. As $J$ diminishes, waves are reflected closer to the surface. The local minimum and maximum of the drag in Fig. 2 correspond to a situation where the reflections occur at altitudes close to the mountain horizontal scale [in dimensional units $\left.\left.z_{T}(1 / L)=\sqrt{J} L\right)\right]$. In these situations, the reflected waves interact destructively and constructively with the emitted waves 
a) Vert. wind, $J=0.10$

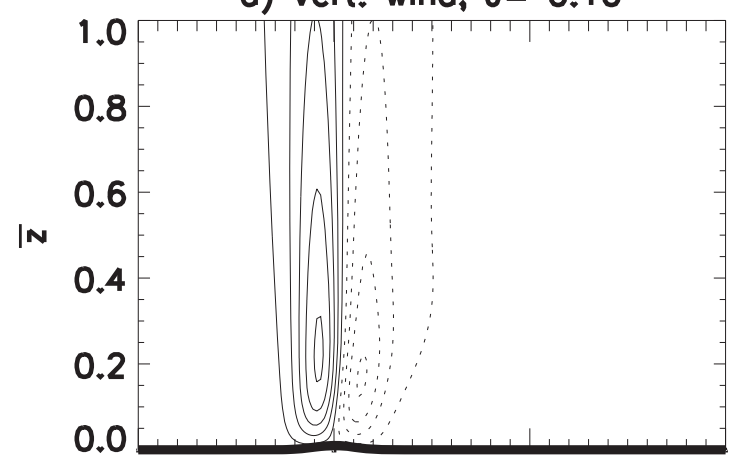

c) Vert. wind, $\mathrm{J}=0.70$

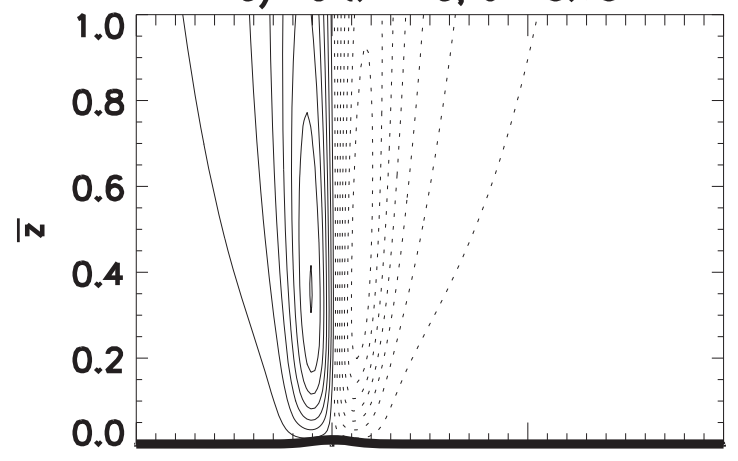

e) Vert, wind, $J=1.70$

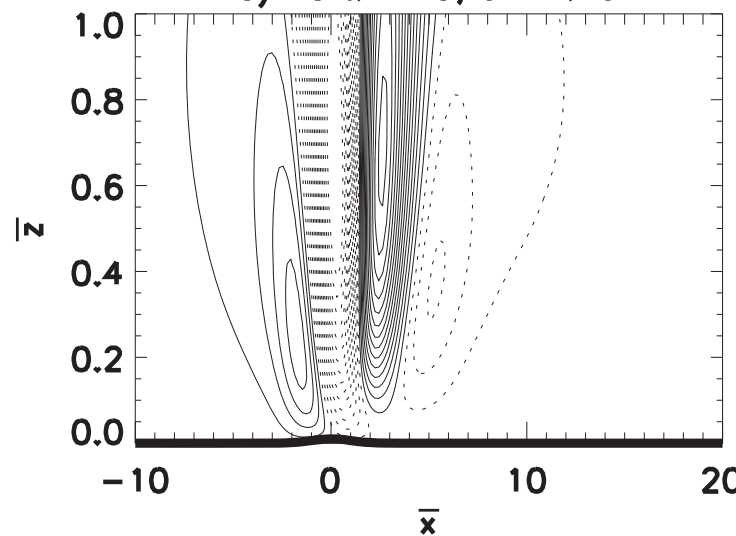

b) Action Flux , $J=0.10$

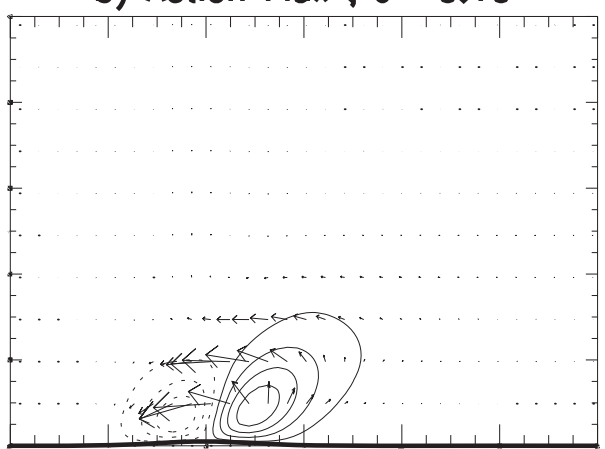

d) Action Flux , $J=0.70$

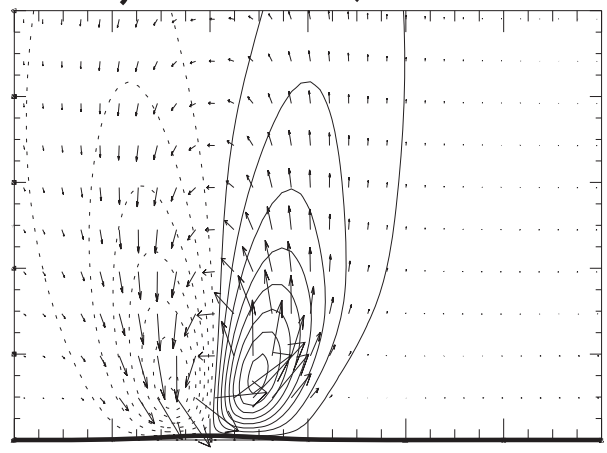

f) Action Flux $1 \uparrow \mathrm{J}=1.70$

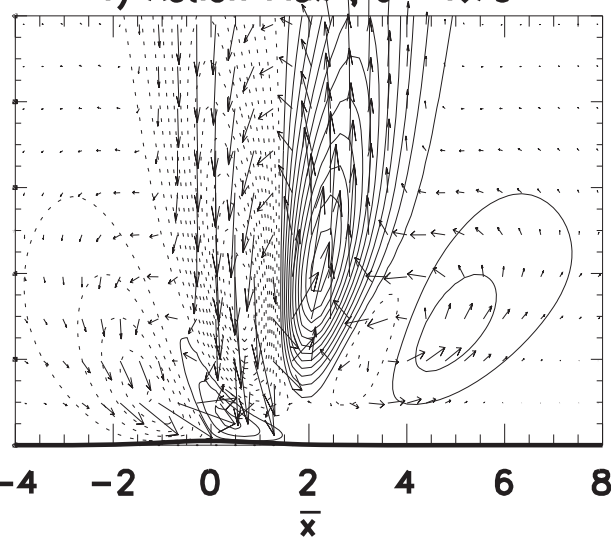

FIG. 3. (a),(c),(e) Vertical velocity and (b),(d),(f) action flux [vertical component $F^{z}$ and vector $\left(F^{x}, F^{z}\right)$ for $S=$ 0.01. Contour interval for $w$ in (a), (c), and (e) is as in Fig. 1b]. Contour interval for vertical component of the wave action flux is as in Fig. 1d.

to produce low-drag and high-drag states, respectively. When the reflections occur higher, the reflected waves return to the surface farther on the lee side, so their effect on the surface pressure becomes small over the hill compared to that of the upward-propagating waves.

\section{Low-drag and high-drag states}

To better appreciate what occurs when the flow is weakly or moderately stratified, we plot in Fig. 3 the vertical velocity and action flux in a weakly stratified case $(J=0.1)$, and in the two moderately stratified cases $(J=0.7$ and $J=$ 1.7) where the drag is respectively lower and larger than the predictor. To ease comparison, we keep all the other parameters similar to those of the reference case (Fig. 1). In the weekly stratified case, the vertical velocity is positive on the upstream side of the ridge and negative on the downstream side. This pattern is similar to the neutral solutions in the inviscid case with no vertical tilt. We also see in Fig. $3 b$ that the wave action flux stays confined inside the inner layer: there is almost no flux of action through the height $z=5 \bar{\delta}(1)$, which measures the inner-layer depth (see Part I). 
a) $\mathrm{J}=0.1$

b) $\mathrm{J}=0.7$

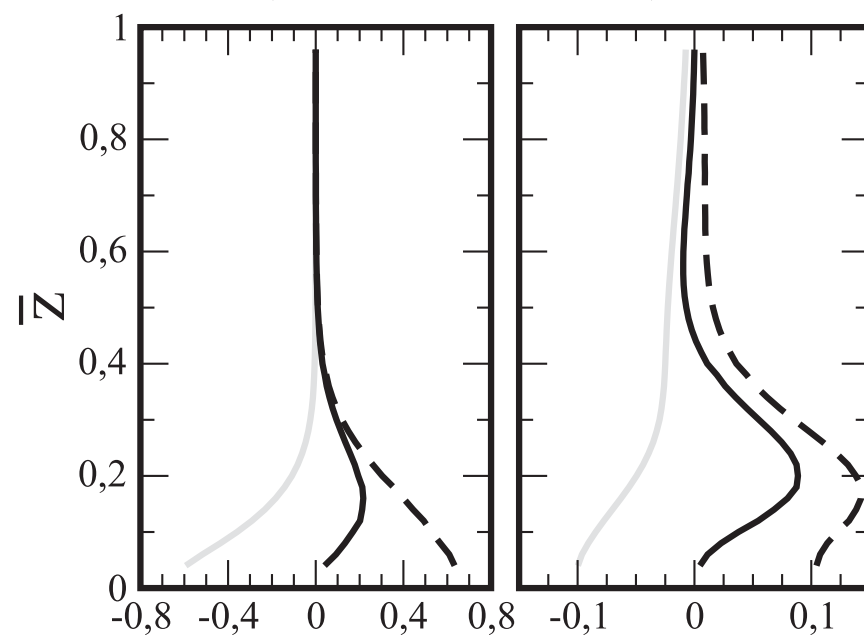

c) $\mathrm{J}=1.6$

d) $\mathrm{J}=4$

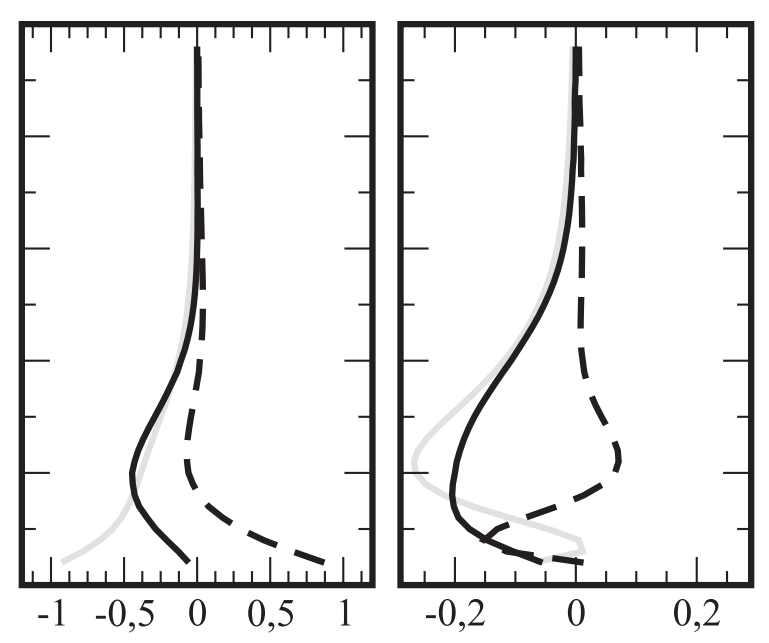

FIG. 4. Vertical profiles of the Reynolds stress (thick line), pressure drag through streamlines (thick gray), and viscous drag through streamlines (thick dashed); see the balance equation [Eq. (27)] and for $S=0.01$ and $\bar{\delta}=0.1$.

We conclude that in the neutral case, the drag cannot have an inviscid wave origin.

For $J=0.7$ in Fig. $3 \mathrm{c}$ one sees that the vertical velocity field has still quite vertical phase lines but it extends significantly higher above the inner layer than in the case with $J=0.1$. Above the inner layer, one sees in Fig. $3 \mathrm{~d}$ that there is substantial pseudomomentum fluxes, pointing upward on the windward side and downward on the leeward side. Although the local directions of pseudomomentum fluxes do not quantify directions of propagation without ambiguity (in theory an action flux is proportional to action times group velocity after averaging over a wave phase), it is quite systematic that for mountain waves a negative vertical component of the wave action flux $\left(F^{z}<0\right)$ indicate upward propagation (although there are variations from one wave crest to the other, as seen in Fig. 1d of Part I). Accordingly, we state that regions above the inner layer where $F^{z}>0$ correspond to downward-propagating waves, as seen in Fig. 3d on the downwind side of the hill. Still in Fig. 3d, we notice that regions with $F^{z}>0$ occupy about the same area as regions with $F^{z}<0$, as if the downward-propagating waves were balancing almost exactly the upward-propagating waves in terms of vertical flux of momentum. This balance probably explains the minimum in pressure drag seen when $J \approx 0.7$ in Fig. 2 .

The case with $J=1.7$ in Fig. 3e presents substantial phase line tilt, and a system of internal waves with two crests and throughs. Upstream and above the ridge, the pseudomomentum flux is quite strong and points downward, as expected for upward-propagating waves. There is also large pseudomomentum flux above the inner layer that points upward but this flux is located well on the downwind side, that is, as if the reflected wave were returning to the surface further downstream than in the case with $J=0.7$. This is again consistent with the fact that the characteristic altitude of the turning points where the waves are reflected $\left(\bar{Z}_{T}(1)=\sqrt{J}\right)$ increases with $J$. Interestingly, it seems that the downward waves in this case return to the surface near enough downstream the mountain to interfere with the surface boundary condition and to produces large pseudomomentum fluxes and drag.

\section{Waves Reynolds stress}

The predictors of the surface pressure drag may not be very useful if we take them as a measure of the effect of the mountain on the large-scale flow, as generally done in mountain meteorology (see discussion in Part I). The reason is that, in a steady state, the wave pseudomomentum flux vector within the inner layer is oriented from the upstream side of the ridge toward the downstream side. This situation differs from the inviscid case where this flux goes through the surface and produces an exchange of momentum between the fluid and the solid ground in the form of a pressure drag. In the hydrostatic case, we concluded that the acceleration that balances the gravity wave drag is not communicated to the Earth surface but rather to the flow below around the inner-layer scale. As we shall see, this is even more problematic in the nonhydrostatic case because mountain drag does not necessarily lead to flow deceleration above the inner-layer scale.

To understand how mountains interact with the large-scale flow, we plot in Fig. 4 the vertical profile of the wave Reynolds stress (in black), the pressure stress (gray) and the viscous stress (dashed) acting along displaced streamlines. These are the three terms of the balance equation derived in Part I:

$$
\overline{\bar{u}} \bar{w}=-\overline{\bar{p}} \partial_{\bar{x}} \bar{\eta}-\bar{\nu} \overline{\left(\bar{\eta} \partial_{\bar{z}}^{2} \bar{u}\right)}, \quad \text { where } \quad \bar{z} \partial_{\bar{x}} \bar{\eta}=\bar{w},
$$

and that can only be estimated above the mountain top $S$. We see in Fig. 4 that at low level, the Reynolds stress is small and there is a balance between pressure and viscous stress. In the inner layer, the magnitude of the Reynolds stress increases with height, reaches an extreme and vanishes when $\bar{z} \rightarrow \infty$ (as expected because all harmonics are evanescent in $\bar{z} \rightarrow \infty$ ). 


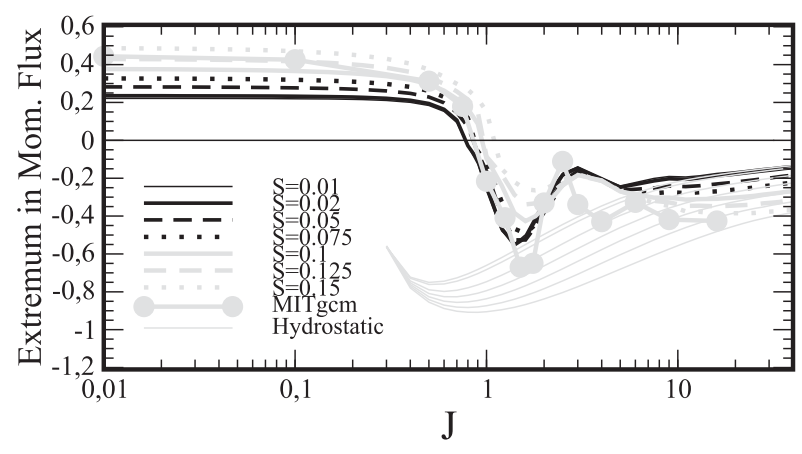

FIG. 5. Extrema in Reynolds stress normalized by the predictor $\mathrm{Dr}_{p}$. Hydrostatic values normalized by $D_{\mathrm{GWP}}$ from Part I are also shown for comparison (thin gray lines). Gray dots are from the MITgcm with $S=0.15$.

What is remarkable is that in the near-neutral case $J=0.1$ as well as in the low-drag case $J=0.7$, the Reynolds stress $\overline{\bar{u}} \bar{w}>0$ is positive in the inner layer such that it should produce a deceleration of the large-scale flow in the lower part of the inner layer [e.g., around $\bar{z} \approx \bar{\delta}(1)$ ] and an acceleration of the largescale flow in the upper part [e.g., around $\bar{z} \approx 3 \bar{\delta}(1)]$. In the stratified case $(J>1)$, we recover the standard result that waves accelerate the large-scale flow in the lower part of the inner layer and decelerate the large-scale flow above, as expected for mountain gravity wave drag (Figs. 4c,d).

It is clear from Fig. 4 that the interesting quantity is the extreme value of the wave Reynolds stress rather than the pressure drag itself. In fact, these extremes are always smaller in amplitude, and even of opposite sign to the pressure drag. We further explore the parameter space, and we plot in Fig. 5 these extremes normalized by the predictor of the pressure drag (26) for different values of the slope and stability. We conclude that our predictors overestimate by a factor of 3 the extreme value of the Reynolds stress and more importantly that the sign of the Reynolds stress extreme changes around $J=1$ : there is flow acceleration above the inner-layer scale $\bar{\delta}$ when $J<1$ and deceleration due to gravity wave drag when $J>$ 1. These acceleration/deceleration are balanced by opposing deceleration/acceleration below $\bar{\delta}(1)$, at least when $S \ll \bar{\delta}(1)$, but these start to be partly transferred to the ground when $S \approx \bar{\delta}(1)$, as in Part I (not shown).

\section{Transition from downstream sheltering to upstream blocking when $S \approx \bar{\delta}$}

To analyze further what occurs in the more nonlinear situations we next consider cases where the slope parameter becomes comparable to the inner-layer scale $\bar{\delta}(1)$. We first consider the upper limit $S=0.185$ beyond which our theoretical model often diverges when $\bar{\delta}(1)=0.1$. We choose $J=0.01$ to illustrate the neutral case and $J=9$ to illustrate the stratified case. We plot in Fig. 6 the streamfunction and the wind field for these two cases as well as in the intermediate case where the reflected waves impact strongly the surface conditions near the mountain $(J=1.7)$. In the near-neutral case (Figs. 6a,b), the wind is intensified on the windward side and small downwind, which correspond to a form of nonseparated sheltering. When stratification increases, this upslope/downslope asymmetry reduces, up to around $J=1$ : the low-drag case with $J \approx 0.7$, for instance, is almost symmetric between the upstream and the downstream side (not shown).

In situations with high $\operatorname{drag}(J=1.7)$, the upslope/downslope asymmetry is not much pronounced, at least on the streamlines in Fig. $6 \mathrm{c}$ near the surface. The most remarkable behavior is the pronounced ridge occurring downstream around $\bar{x}=4$, which corresponds to the strong positive vertical wind anomaly already present in the case with small slope and around the same place (Fig. 3e). This pronounced oscillation cannot be attributed to trapped lee waves because these waves are not present in our configuration: trapped waves are always related to neutral modes of Kelvin-Helmholtz (KH) instability when the wind vanishes at the surface (Lott 2016), and these modes do not exist when the Richardson number is constant according the Miles-Howard theorem (Miles 1961; Howard 1961). The absence of trapped modes differs from the study of Keller (1994), who first solved the Bessel's equation to analyze inviscid trapped waves in constant shear cases. In Keller (1994) nevertheless, the wind at the surface is nonzero. Lott (2016) proposes that when the surface wind does not vanish, the surface wind shear is infinite and the surface Richardson number is null, so downward-propagating stationary waves can be entirely reflected and neutral modes can exist.

In situations with strong stratification $(J=9$, Figs. $6 \mathrm{e}, \mathrm{f})$, we recover the upstream blocking and downslope winds present in the hydrostatic case in Part I, although in this case all the waves are reflected toward the ground. We do not discuss the results from the MITgcm, but we have used this model in all the configurations with $S=0.15$ and $S=0.185$ presented in this paper and the solutions from the nonlinear model are almost identical to those shown in Fig. 6 (see also the thorough comparison in Part I, where the validation of the theory by the model was excellent).

We propose one last index to characterize the downstream sheltering versus upstream blocking as a function of $S$ and $J$. We define this index as the ratio between the wind amplitude along the downwind slope and the upwind slope of the ridge defined as

$$
\frac{\underbrace{\max }_{\bar{z}<2 \bar{h} / 3,0<\bar{x}<2} \sqrt{(\bar{z}+\bar{u})^{2}+\bar{w}^{2}}}{\underbrace{\max }_{\bar{z}<2 \bar{h} / 3,-2<\bar{x}<0} \sqrt{(\bar{z}+\bar{u})^{2}+\bar{w}^{2}}},
$$

and we plot this index for several values of $J$ and $S$ in Fig. 7. As in the hydrostatic case and for large values of $J$, this index can easily reach values around 4 or 5 for slopes near the inner-layer depth and larger. This ratio is always around 1 when $J \approx 1$, as in the hydrostatic case, except near the critical value $J=1.7$, which corresponds to the high-drag scenario. For $J<1$, the ratio becomes smaller than 1 , which corresponds to nonseparated sheltering. The smallest values we obtain are around 0.5 for $J=0.01$ and slopes $S \approx 0.15$. 


\section{a) Stream fonction, $J=0.01, S=0.185$ b) Wind vector, $J=0.01, S=0.185$}

|N
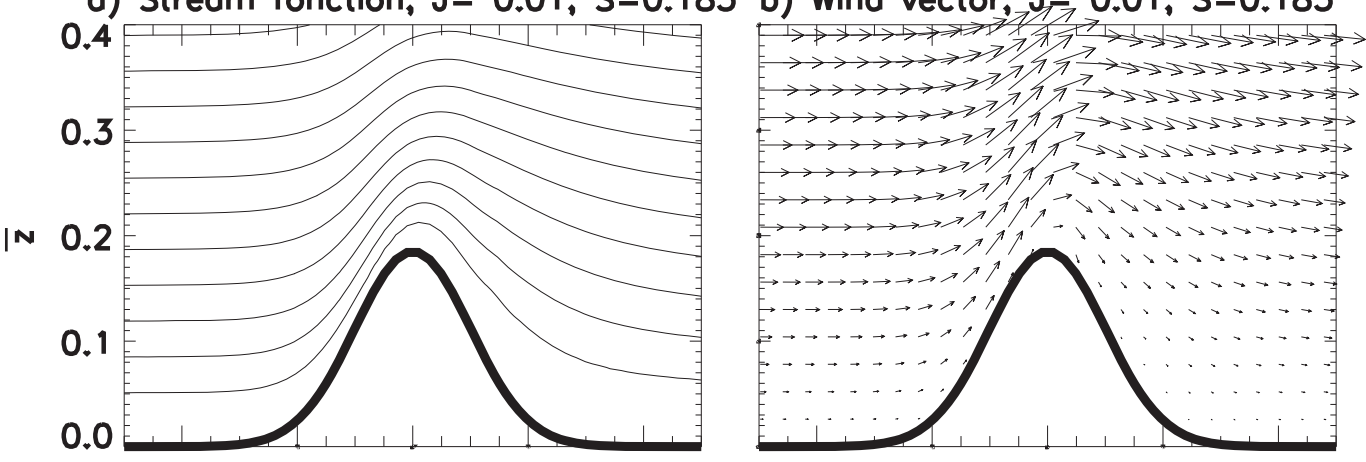

c) Stream fonction, $J=1.70, S=0.185$
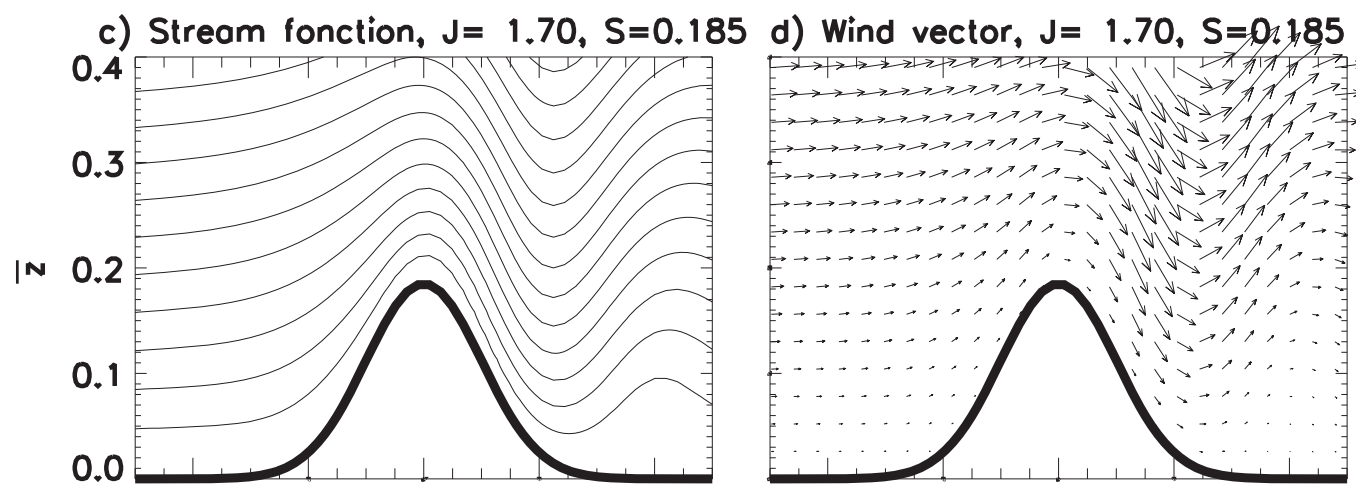

e) Stream fonction, $J=9.00, S=0.185$
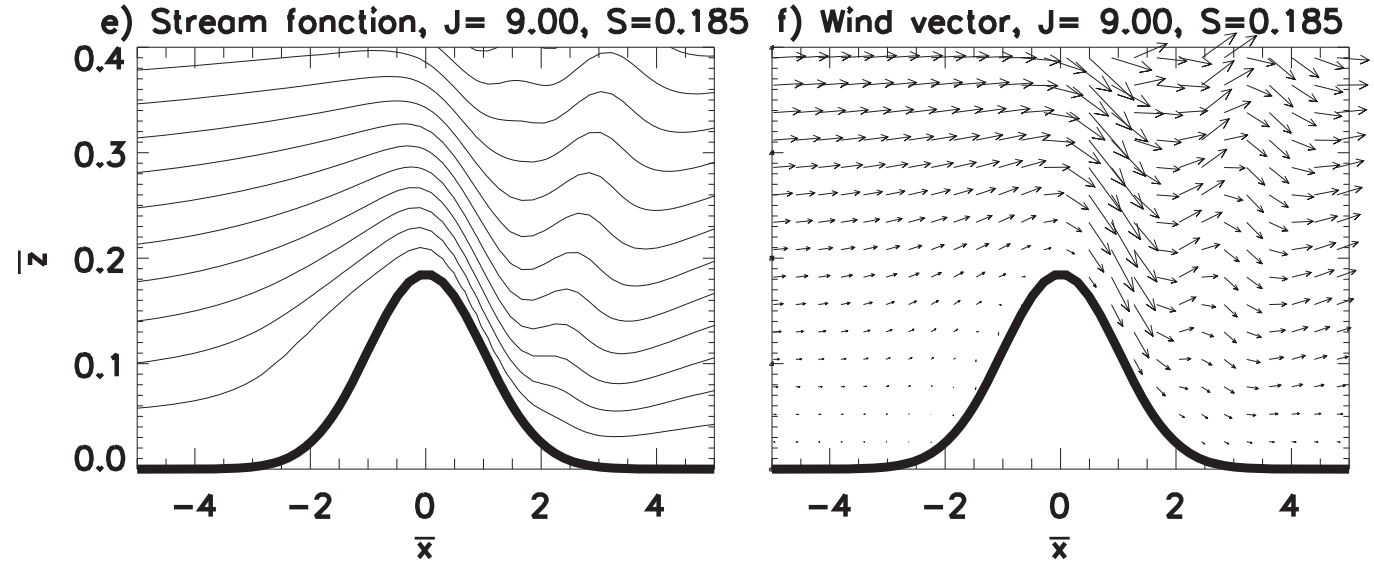

FIG. 6. (a),(c),(e) Streamfunction defined by $\partial \bar{\psi} / \partial \bar{z}=\bar{u}+\bar{z}$. (b),(d),(f) Total wind. In all panels $S=0.185$ and $\bar{\delta}=0.1$. In (a) and (b) $J=0.01$, in (c) and (d) $J=1.70$, and in (e) and (f) $J=9.00$.

\section{Conclusions}

\section{a. Summary}

The first result of our nonhydrostatic analysis confirm that of Part I: the pressure drag in the stratified case is a wave drag to be computed at the inner-layer scale. We extend it to the neutral case, replacing the wave drag by a form drag that is also predictable. We also show that the transition between the stratified and neutral case is well captured by the Richardson number and occurs around $J=1$ [see Eq. (26)]. More precisely, the wave drag predictor is well adapted to cases with $J>2$, the form drag predictor is well adapted for $J<0.5$. A quite rich transition occurs between $0.5<J<2$, and when the reflected waves deeply affect the surface condition producing low-drag state and high-drag states at $J \approx 0.7$ and $J \approx 1.7$, respectively (see Fig. 4).

Differences with Part I are more pronounced when we analyze the vertical distribution of the Reynolds stress. In the stratified case $(J>2)$, the low-level confinement of the waves due to the nonhydrostatic terms make that there is no gravity wave drag in the far field by construction. As we see in Fig. 4d the wave drag is then deposited in the upper part of the inner layer [roughly between $2 \bar{\delta}(1)<\bar{z}<5 \bar{\delta}(1)$ ], the maximum of the Reynolds stress staying well predicted by the hydrostatic 


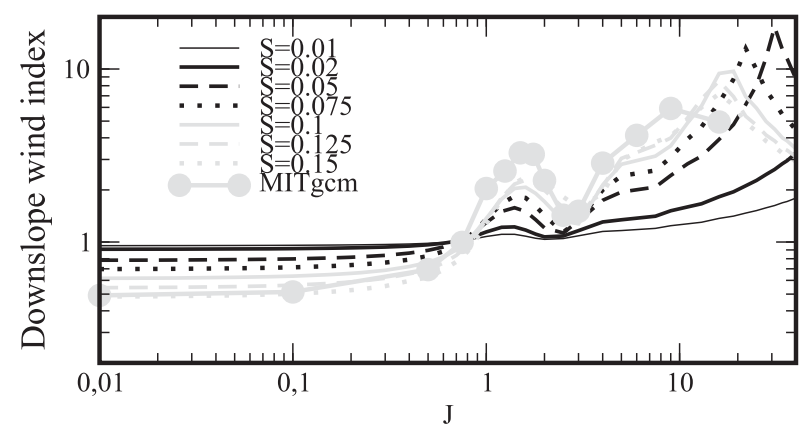

FIG. 7. Downslope sheltering vs upstream blocking index defined as the ratio between the max downslope wind amplitude and the max upslope wind amplitude [see Eq. (28)]. Gray dots are from the MITgcm with $S=0.15$.

theory. As in Part I, a fraction of the drag is extracted from the lower part of the inner layer. In the more neutral cases when $J<0.5$ we find an opposite behavior, the Reynolds stress is positive in the inner layer and so accelerates the large-scale flow in the upper part of the inner layer, and decelerates the flow in the lower part of the inner layer (see Fig. 4a).

Our results also indicate how the mountain modify the flow around the obstacle when its height is comparable to the innerlayer scale $[S \approx \bar{\delta}(1)]$. At large $J \gg 1$, the flow on the upstream side is blocked, whereas the downslope winds are substantial in the inner layer on the lee side, again as in Part I. At small $J \ll 1$ again, an opposite behavior is found, the obstacle produces a region of calm flow on the lee side, while the flow is accelerated along the upstream side, this is a form of nonseparated sheltering. The high-drag state is characterized by a strong and unique wave crest downstream near the top of the inner layer, illustrating without ambiguity that a strong lee-wave crest well downstream of the mountain is not necessarily associated with the presence of resonant trapped modes (see discussion in section 6).

\section{b. Discussion}

One motivation of this article is that state-of-the-art numerical weather prediction and climate models parameterize subgrid-scale orography (SSO) in the neutral case using techniques derived from boundary layer parameterization schemes (Beljaars et al. 2004) and treat the stratified cases separately and using low-level wave drag schemes (Lott and Miller 1997). The choice of one parameterization versus the other is enforced by ad hoc criteria (see introduction in Part I), so it seems worthwhile revisiting the criteria for the transition between the two regimes. Moreover, the standard model resolution of atmospheric models is such that we are today in a gray zone between resolved and unresolved mesoscale orographic flows (Vosper et al. 2016), and that this gray zone is at scales near the transition scales between neutral and stratified conditions. In this context, we demonstrated that the drag (wave drag or form drag) can be predicted using linear theory and using flow parameters evaluated at the inner-layer scale. For instance, and if one wish to apply Eq. (26) in a large-scale model with parameterized turbulent drag, one should evaluate the altitude at which the disturbance in turbulent drag equals the advection of the disturbance in wind and calculate the background parameters of interest (incident wind, stratification, and dissipation) at this altitude. To take a simple illustration, let us consider that the model in question use eddy diffusivities based on mixing length theory,

$$
\nu=\Lambda^{2}\left\|\frac{d u_{0}}{d z}\right\|, \quad \text { with } \quad \frac{1}{\Lambda}=\frac{1}{\kappa z}+\frac{1}{\lambda},
$$

where $\Lambda$ is the mixing length of the undisturbed flow and $\kappa$ the von Kármán constant. The inner-layer scale $\delta$ at which advection equilibrates turbulent drag in the equation for the disturbance wind is

$$
\frac{u_{0}(\delta)}{L}=2 \frac{\Lambda^{2}}{\delta^{2}} \frac{d u_{0}}{d z}(\delta)
$$

To adapt such an equation to realistic conditions, the background flow could be measured in the dominant direction of the synoptic winds, and the characteristic scale $L$ evaluated from SSO parameters in this direction. Once done, and if there is a need to consider real 3D mountains, a linear wave theory could be used to predict the drag from Fourier analysis of the SSO over the grid of interest. Note nevertheless that these are just directions that could be worth testing. One could also reverse the analysis and use our neutral versus stratified flow criteria to evaluate the scale $L$ above which gravity waves should be parameterized and below which boundary layer effects should be parameterized.

Other aspects could be worth to try to include in large-scale models. For instance, we show in the stratified case $(J>1)$ that the wave drag is deposited around the top of the inner layer rather than below turning altitudes. This is to be contrasted with papers in which trapped waves are not dissipated (basically in the absence of surface critical levels here), and where the wave Reynolds stress decays with altitude up to the turning heights, and to balance a downstream horizontal flux of pseudomomentum Georgelin and Lott (2001). An important difference with Georgelin and Lott (2001) is that our solutions do not include pure trapped waves (see discussion in section 6). As we shall see in Part III, when such modes are present, the depth of the inner layer will still be that over which the wave drag is redistributed once the waves are all dissipated. This questions the way low-level drag due to trapped lee waves should be parameterized in models (Teixeira et al. 2013).

Another aspect worth noticing is the flow deceleration at low levels seen in the neutral case and that is equilibrated by a flow acceleration at the top of the inner layer. It is strongly reminiscent of the concept of envelope orography introduced by Wallace et al. (1983), where SSO is not necessarily represented by pure drag forces but rather by forces that higher up the lower bound of the model without necessarily decelerating the large-scale flow (Lott 1999). This low-level deceleration and high-level acceleration are the opposite of what occurs in stable cases where the stress is due to gravity waves.

\section{c. Limitations}

A first limit of our results is that they assume linear fields above the surface and small slopes, so it could be argued that 
they cannot be applied in the context of parameterization of real mountains. To moderate such critics, we can recall that we have tried to extend our calculation up to the limit where the mountain height and the inner-layer height compare. We can also recall that parameterizations are always based on linear theories, and are then adapted to nonlinear configurations by comparing the vertical scales of disturbances and the height of the obstacles (criteria that always involve the parameters $J$ and $S$ that we use here). In all these parameterizations, the linear values are always upper bounds of the drag. Interestingly, linear theories are also used to predict these bounds, essentially via their prediction of the separation points (Smith 1989; Lott and Miller 1997; Ambaum and Marshall 2005). In this context, the present article enforces the point that linear theories can be used to predict nonlinear fields, since here a linear theory with nonlinear boundary condition accurately reproduce the sheltering and the blocking occurring in the more nonlinear cases. Also, and this is maybe a significant point, it is worth recalling that with increasing horizontal resolution, the height of subgrid-scale mountains decreases so they are more and more located within the boundary layer, maybe rendering our linear dissipative formalism more and more adapted.

A second limit is that our calculations use eddy diffusivities that are constant whereas in reality they strongly depend on altitude and decrease near the surface [this is evident in the mixing length model; see (29)]. This decrease in diffusivity also makes that the background flow tends to have logarithmic profiles on $u_{0}$ and $\rho_{0}$ near the surface. With these profiles the background Richardson number is null at the surface, a situation that does not produce strong critical-level absorptions in the inviscid limit. Accordingly, all our results overstate the absorption of the waves when they return to the surface. The logarithmic profiles also introduce background flow curvatures that play a central role in Belcher and Wood (1996), since they define the midlayer height at which the flow parameters should be evaluated to calculate the drag and the transition between the neutral and stratified case [see discussion of Eq. (1) in section 1]. These curvatures also potentially yield resonant mode that can develop in trapped lee waves (Lott 2007, 2016). These aspects will be developed in subsequent papers.

Acknowledgments. This work was supported by the Laboratoire de Recherche Conventionné "Yves Rocard," a collaborative unit between CEA and Ecole Normale Supérieure.

\section{REFERENCES}

Abramowitz, M. J., and I. A. Stegun, 1964: Handbook of Mathematical Functions. 9th ed. Dover Publication, 1045 pp.

Ambaum, M., and D. Marshall, 2005: The effects of stratification on flow separation. J. Atmos. Sci., 62, 2618-2625, https:// doi.org/10.1175/JAS3485.1.

Athanassiadou, M., 2003: Wave and form drag: Their relation in the linear gravity wave regime. Tellus, 55A, 173-180, https:// doi.org/10.3402/tellusa.v55i2.12090.

Belcher, S. E., and N. Wood, 1996: Form and wave drag due to stably stratified turbulent flow over low ridges. Quart. J. Roy. Meteor. Soc., 122, 863-902, https://doi.org/10.1002/ qj.49712253205.
Beljaars, A. C. M., A. R. Brown, and N. Wood, 2004: A new parametrization of turbulent orographic form drag. Quart. J. Roy. Meteor. Soc., 130, 1327-1347, https://doi.org/10.1256/ qj.03.73.

Doyle, J. D., and D. R. Durran, 2002: The dynamics of mountainwave-induced rotors. J. Atmos. Sci., 59, 186-201, https://doi.org/ 10.1175/1520-0469(2002)059<0186:TDOMWI >2.0.CO;2.

Durran, D. R., 1990: Mountain waves and downslope winds. Atmospheric Processes over Complex Terrain, Meteor. Monogr., No. 23, Amer. Meter. Soc., 59-83.

Georgelin, M., and F. Lott, 2001: On the transfer of momentum by trapped lee waves: Case of the IOP3 of PYREX. J. Atmos. Sci., 58, 3563-3580, https://doi.org/10.1175/1520-0469(2001) 058<3563:OTTOMB $>2.0 . \mathrm{CO} ; 2$.

Howard, L. N., 1961: Note on a paper of John W. Miles. J. Fluid Mech., 10, 509-512, https://doi.org/10.1017/S0022112061000317.

Hunt, J. C. R., S. Leibovich, and K. J. Richards, 1988: Turbulent shear flows over low hills. Quart. J. Roy. Meteor. Soc., 114, 1435-1470, https://doi.org/10.1002/QJ.49711448405.

Keller, T. L., 1994: Implications of the hydrostatic assumption on atmospheric gravity waves. J. Atmos. Sci., 51, 1915-1929, https:// doi.org/10.1175/1520-0469(1994)051<1915:IOTHAO > 2.0.CO;2.

Lapworth, A., and S. Osborne, 2019: Gravity-wave drag in the stable boundary layer over moderate terrain. Bound.-Layer Meteor., 171, 175-189, https://doi.org/10.1007/S10546-01800422-3.

Lott, F., 1999: Alleviation of stationary biases in a GCM through a mountain drag parameterization scheme and a simple representation of mountain lift forces. Mon. Wea. Rev., 127, 788-801, https://doi.org/10.1175/1520-0493(1999) $127<0788$ :AOSBIA $>2.0 . \mathrm{CO} ; 2$.

_ 2007: The reflection of a stationary gravity wave by a viscous boundary layer. J. Atmos. Sci., 139, 3363-3371, https://doi.org/ 10.1175/JAS4020.1.

— 2016: A new theory for downslope windstorms and trapped lee waves. J. Atmos. Sci., 73, 3585-3597, https://doi.org/ 10.1175/JAS-D-15-0342.1.

— parameterization: Its testing in the ECMWF model. Quart. J. Roy. Meteor. Soc., 123, 101-127, https://doi.org/10.1002/ QJ.49712353704.

- B. Deremble, and C. Soufflet, 2020: Mountain waves produced by a stratified shear flow with a boundary layer. Part I: Hydrostatic case. J. Atmos. Sci., 77, 1683-1697, https://doi.org/ 10.1175/JAS-D-19-0257.1.

Marshall, J., A. Adcroft, C. Hill, L. Perelman, and C. Heisey, 1997: A finite-volume, incompressible Navier Stokes model for studies of the ocean on parallel computers. J. Geophys. Res., 102, 5753-5766, https://doi.org/10.1029/96JC02775.

Miles, J. W., 1961: On the stability of heterogeneous shear flow. J. Fluid Mech., 10, 496-508, https://doi.org/10.1017/S0022112061000305.

Pokharel, B., B. Geerts, X. Chu, and P. Bergmaier, 2017: Profiling radar observations and numerical simulations of a downslope wind storm and rotor on the lee of the medicine bow mountains in Wyoming. Atmosphere, 8, 39, https://doi.org/10.3390/ ATMOS8020039.

Reinert, D., V. Wirth, J. Eichhorn, and W.-G. Panhans, 2007: A new large-eddy simulation model for simulating air flow and warm clouds above highly complex terrain. Part I: The dry model. Bound.-Layer Meteor., 125, 109-132, https://doi.org/ 10.1007/S10546-007-9183-8.

Sachsperger, J., S. Serafin, and V. Grubisic, 2016: Dynamics of rotor formation in uniformly stratified two-dimensional flow 
over a mountain. Quart. J. Roy. Meteor. Soc., 142, 1201-1212, https://doi.org/10.1002/QJ.2746.

Serafin, S., and Coauthors, 2018: Exchange processes in the atmospheric boundary layer over mountainous terrain. Atmosphere, 9, 102, https://doi.org/10.3390/atmos9030102.

Smith, F., 1973: Laminar flow over a small hump on a flat plate. J. Fluid Mech., 57, 803-824, https://doi.org/10.1017/S002211207300203X.

Smith, R. B., 1989: Mountain-induced stagnation points in hydrostatic flow. Tellus, 41A, 270-274, https://doi.org/10.1111/ J.1600-0870.1989.TB00381.X.

— , Q. Jiang, and J. D. Doyle, 2006: A theory of gravity wave absorption by a boundary layer. J. Atmos. Sci., 63, 774-781, https://doi.org/10.1175/JAS3631.1.

Sykes, R., 1978:Stratification effects in boundary layer flow over hills. Proc. Roy. Soc. London, A361, 225-243, https://doi.org/ 10.1098/rspa.1978.0100.

Teixeira, M. A., J. L. Argain, and P. M. A. Miranda, 2013: Orographic drag associated with lee waves trapped at an inversion. J. Atmos. Sci., 70, 2930-2947, https://doi.org/10.1175/JAS-D-12-0350.1.
Tsiringakis, A., G. Steeneveld, and A. Holtslag, 2017: Small-scale orographic gravity wave drag in stable boundary layers and its impact on synoptic systems and near-surface meteorology. Quart. J. Roy. Meteor. Soc., 143, 1504-1516, https://doi.org/ 10.1002/QJ.3021.

Voigt, M., and V. Wirth, 2013: Mechanisms of banner cloud formation. J. Atmos. Sci., 70, 3631-3640, https://doi.org/10.1175/ JAS-D-12-0353.1.

Vosper, S. B., A. Brown, and S. Webster, 2016: Orographic drag on islands in the NWP mountain grey zone. Quart. J. Roy. Meteor. Soc., 142, 3128-3137, https://doi.org/10.1002/QJ.2894.

Wallace, J. M., S. Tibaldi, and A. J. Simmons, 1983: Reduction of systematic forecast errors in the ECMWF model through the introduction of an envelope orography. Quart. J. Roy. Meteor. Soc., 109, 683-717, https://doi.org/10.1002/QJ.49710946202.

Weng, W., 1997: Stably stratified boundary-layer flow over low hills: A comparison of model results and field data. Bound.-Layer Meteor., 85, 223-241, https://doi.org/10.1023/ A:1000441917023. 